\title{
Endometriose Cutânea Primária: Diagnóstico Raro e Desafiador
}

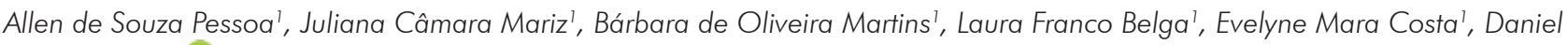 \\ Lago Obadia' \\ 'Departamento de Dermatologia, Hospital Universitário Pedro Ernesto, Universidade do Estado do Rio de Janeiro, Rio de Janeiro, \\ Brasil
}

RESUMO - A endometriose ocorre em cerca de 10\% das mulheres em idade reprodutiva, é histopatologicamente definida pela presença de tecido glandular endometrial e/ou estroma fora da cavidade uterina. A principal localização cutânea é o umbigo. A endometriose cutânea primária é rara e subdiagnosticada. Deve ser suspeitada na presença de nódulo eritemato-violáceo com tendência a sangramento, especialmente durante o período menstrual, mesmo na ausência de cirurgia abdominal anterior ou história de endometriose pélvica. A ressecção cirúrgica é o tratamento de escolha. A avaliação ginecológica é recomendada, visto que a associação com a doença pélvica ocorre em $26 \%$ dos casos de endometriose cutânea. Relatamos caso de paciente sem história de cirurgias abdominais e/ou endometriose, com nódulo violáceo em cicatriz umbilical com um ano de evolução, com sangramento durante o período menstrual e após traumas.

PALAVRAS-CHAVE - Doenças da Pele; Endometriose; Umbigo.

\section{Primary Cutaneous Endometriosis: Rare and Challenging Diagnosis}

ABSTRACT - Endometriosis occurs in about 10\% of women of reproductive age. It is histopathologically characterized by the presence of endometrial glandular tissue and/or stroma outside the uterine cavity. The main cutaneous site is the umbilicus. Primary skin endometriosis is rare and underdiagnosed. It should be suspected in the presence of an erythemato-violaceous nodule with a bleeding tendency, especially during the menstrual period, even in the absence of previous abdominal surgery or history of pelvic endometriosis. Surgical resection is the treatment of choice. Gynecological evaluation is recommended, since the association with pelvic disease occurs in $26 \%$ of cases of skin endometriosis. We report a case of a violaceous nodule on an umbilical scar showing bleeding during menstruation, which evolved over a year following trauma, in a patient with no history of abdominal surgeries and/ or endometriosis.

KEYWORDS - Endometriosis; Skin Diseases; Umbilicus.

\section{INTRODUÇÃO}

A endometriose, doença estrogénio-dependente ${ }^{1,2}$ inicialmente descrita por Rokitansky em 1860, 1,3 acomete cerca de $10 \%$ das mulheres em idade reprodutiva. ${ }^{2,4,5}$ Histopatologicamente é definida pela presença de tecido glandular endometrial e/ou estroma fora da cavidade uterina que pode ocorrer por transformação metaplásica, metástase ou iatrogenia., ${ }^{1,3}$ Os sintomas mais frequentes incluem dor pélvica, dispareunia e dismenorreia, mas variam consoante a localização dos implantes endometriais podendo ser assintomática em $50 \%$ dos casos. ${ }^{1}$ É causa conhecida de infertilidade e tem como localização mais frequente os órgãos pélvicos, principalmente os ovários e trompas uterinas. ${ }^{1-3}$

Em cerca de $12 \%$ dos casos de endometriose são encontradas lesões extragenitais, que ocorrem em qualquer órgão, incluindo cérebro, pulmão, fígado, mesentério, intestino, pleura e pele. ${ }^{2,3,5} \mathrm{~A}$ endometriose cutânea é um
Correspondência: Bárbara de Oliveira Martins

Dept Dermatologia/Universidade do Estado do Rio de Janeiro

Rua Visconde de Pirajá, 180/403, Ipanema

CEP 22410-000 - Rio de Janeiro, Brasil

E-mail: bomartins1@gmail.com

DOI: https://dx.doi.org/10.29021/spdv.78.4.1275
Recebido/Received 2019/09/11

(c) Autor (es) (ou seu (s) empregador (es)) 2020 Revista SPDV. Reutilização permitida de acordo com CC BY-NC. Nenhuma reutilização comercial.

(C) Author(s) (or their employer(s)) 2020 SPDV Journal. Re-use permitted under CC BY-NC. No commercial re-use. 


\section{Caso Clínico}

evento raro, ${ }^{3}$ representando $5,5 \%$ dos casos de endometriose extrapélvica. ${ }^{6} \mathrm{~A}$ sua patogênese ainda não está bem definida, ${ }^{2}$ permanecendo como um desafio diagnóstico. ${ }^{6}$

\section{CASO CLÍNICO}

Paciente feminina, 31 anos, nulípara, apresentava nódulo violáceo na cicatriz umbilical há 1 ano, medindo cerca de $0,5 \mathrm{~cm}$ de diâmetro (Fig. 1). Relatava sangramento espontâneo coincidente com o período menstrual e aos mínimos traumas. Negava doenças ginecológicas e procedimentos cirúrgicos abdominais ou pélvicos prévios. Foi feita a excisão cirúrgica da lesão, com incisão fusiforme perpendicular à linha alba. A sutura simples foi realizada com mononylon 4-0. Ao exame histopatológico, foram observadas estruturas glandulares dilatadas no meio de estroma celular do tipo endometrial e infiltrado linfocítico perivascular (Fig.s 2 e 3), achados compatíveis com endometriose cutânea. Avaliação ginecológica descartou endometriose pélvica. A paciente evoluiu com resultado estético satisfatório, e não houve sinais de recidiva da doença após dois anos de seguimento clínico.

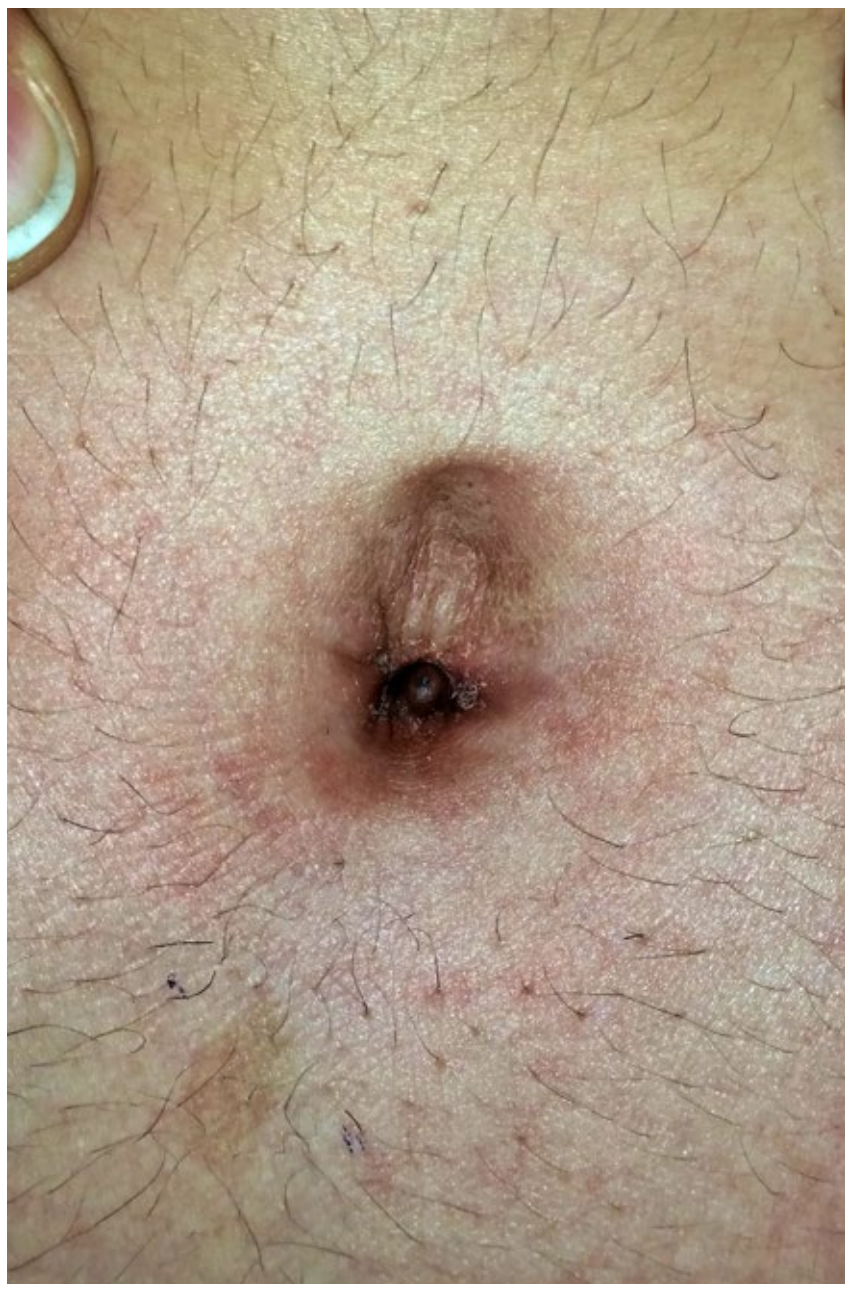

Figura 1 - Nódulo violáceo medindo 0,5cm de diâmetro na cicatriz umbilical com 1 ano de evolução.

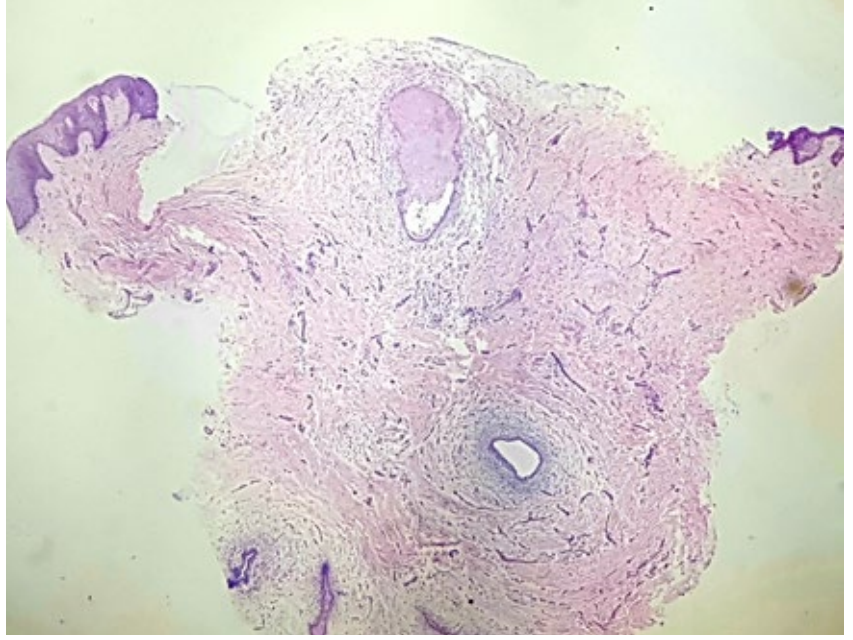

Figura 2 - Estruturas glandulares dilatadas em meio a estroma celular do tipo endometrial e infiltrado linfocítico perivascular (H\&E, 100x).

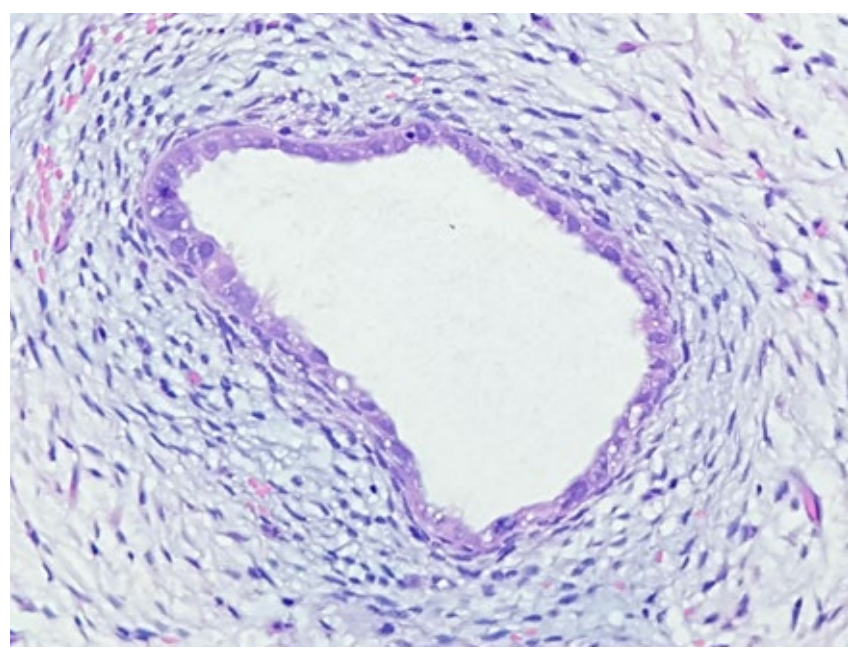

Figura 3 - Detalhe da estrutura glandular endometrial imersa em estroma frouxo mixóide (H\&E, 400x).

\section{DISCUSSÃO}

A endometriose cutânea acomete mulheres sobretudo entre os 30 a 35 anos $^{6}$ e tem como localização principal o umbigo $^{5}$ - acredita-se que seja pelo fato do seu funcionamento como cicatriz fisiológica. ${ }^{6}$ A maioria dos casos (70\%) são secundários à cirurgia pélvica anterior como cesariana - que aumenta o risco em 27 vezes -, exérese de cisto de glândula de Bartholin, injeções intrauterinas para abortamento, laparoscopia, amniocentese, ligação tubária, episiotomia, além de apendicectomia e hernioplastia inguinal ou abdominal. 1,3,6 A endometriose cutânea surge cerca de 30 meses após estes procedimentos cirúrgicos. ${ }^{6} \mathrm{Em} 0,5 \%$ a $1 \%$ de todos os casos não houve cirurgia prévia - trata-se de endometriose primária ou espontânea, ${ }^{2,6}$ como no presente caso. É também chamada nódulo de Villar - médico que primeiro descreveu em 1886.6,7 
A lesão cutânea tem dimensão média de 2 a $3 \mathrm{~cm}$ e pode ter apresentação clínica variável, com coloração vermelha, azulada, roxa ou acastanhada, dependendo da profundidade e quantidade de hemorragia, e é circundada por eritema. ${ }^{1,2,6,7}$ A endometriose cutânea pode ser assintomática em $80 \%$ dos casos, ou cursar com edema, dor e sangramento associados ao ciclo menstrual. ${ }^{6}$ É uma patologia subdiagnosticada, havendo uma diversidade de diagnósticos diferenciais, que incluem hérnia, persistência/lesão do úraco, granulomas, melanoma, abscesso, fístula, nevo benigno, lipoma, queloide, nódulo de Irmã Maria José (metástase cutânea umbilical). 2,6-9

A patogénese da endometriose umbilical ainda não está bem esclarecida, ${ }^{2,8}$ a teoria mais aceita é a que sugere a migração de tecido endometrial a partir da menstruação retrógrada, havendo, então a implantação das células endometriais na superfície peritoneal dos órgãos pélvicos. ${ }^{2}$ Há, ainda, a descrição da migração linfática ou vascular, metaplasia celular, cujo processo envolve transformação de células mesoteliais do peritoneu de origem celômica em células endometriais; e a metástase iatrogénica, associada a disseminação de células endometriais a partir de procedimentos cirúrgicos, com implantação das células na cicatriz. ${ }^{1,2,6}$ No que se refere a endometriose umbilical isolada, o acometimento pode ser em virtude da transformação metaplásica de remanescentes do úraco ou, ainda, advindo da contaminação do cordão umbilical por células endometriais após o nascimento. ${ }^{2}$

A investigação é baseada na anamnese, exame físico detalhados e no exame histopatológico. ${ }^{6,7}$ A dermatoscopia ao evidenciar coloração avermelhada homogénea com pequenas estruturas globulares vermelhas pode contribuir para o diagnóstico. ${ }^{6,7}$ A punção por agulha fina guiada por ultrassom é um método controverso, uma vez que tem precisão variável e há o risco de implantação de tecido endometrial além do sítio de punção e é inadequada para exclusão de malignidade. ${ }^{2,6}$ Assim a confirmação diagnóstica está dependente do exame histopatológico. ${ }^{2,6} \mathrm{O}$ estudo da endometriose cutânea deve ser complementado com avaliação ginecológica e exames de imagem como ultrassonografia pélvica/abdominal, ressonância magnética e tomografia computadorizada, ${ }^{1,2,6,8}$ para avaliar outras lesões intra-abdominais que ocorrem em $26 \%$ dos casos de endometriose cutânea. ${ }^{3}$

O tratamento de escolha é a ressecção cirúrgica. ${ }^{2,6,9}$ Não há técnica estabelecida em virtude da raridade desta patologia, podendo ser realizada mediante excisão completa do umbigo com ou sem reconstrução da fáscia subjacente e peritoneu ou remoção apenas do nódulo endometrial, poupando o umbigo, como no presente caso. ${ }^{10}$ A terapia hormonal é sugerida por alguns autores como forma de reduzir o tamanho da lesão e aliviar sintomas, no entanto, o uso de análogos da hormona libertadora de gonadotrofinas, danazol ou anticoncepcional hormonal oral ${ }^{2,8,9,10}$ no pré-operatório pode levar a excisão incompleta com recorrência. ${ }^{2,6} \mathrm{~A}$ recorrência é rara, 9 mas pode acontecer nos 2 anos que se seguem à excisão inicial. ${ }^{10}$

$\mathrm{Na}$ literatura, está bem descrito, apesar de rara, a possibilidade de transformação maligna a partir de um endometrioma umbilical, ${ }^{1-3}$ o que corrobora a escolha da ressecção cirúrgica como melhor opção terapêutica e a necessidade de uma exame histopatológico. 3,7 A aspiração por agulha fina com exame citológico pode levar a resultados falso-positivos para malignidade pela identificação de atipias nas células glandulares. ${ }^{3}$

\section{CONCLUSÃO}

A endometriose umbilical primária é uma patologia extremamente rara e frequentemente subdiagnosticada, gerando múltiplas idas a diversos médicos e atraso no diagnóstico, podendo levar de 9 a 12 anos entre o início dos sintomas e a confirmação diagnóstica para todos os tipos de endometriose. ${ }^{4}$ Sendo assim, é importante manter alta suspeição diagnóstica diante de um paciente com massa palpável na parede abdominal, mesmo sem história de cirurgia prévia, atentando para os sintomas associados ao ciclo menstrual. 2,6 A condução do caso deve envolver uma equipe multidisciplinar composta por cirurgião geral, radiologista, dermatologista e ginecologista. ${ }^{2}$

Conflitos de interesse: Os autores declaram a inexistência de conflitos de interesse na realização do presente trabalho.

Fontes de financiamento: Não existiram fontes externas de financiamento para a realização deste artigo.

Confidencialidade dos dados: Os autores declaram ter seguido os protocolos da sua instituição acerca da publicação dos dados de doentes.

Consentimento: Consentimento do doente para publicação obtido.

Proveniência e revisão por pares: Não comissionado; revisão externa por pares.

Conflicts of interest: The authors have no conflicts of interest to declare.

Financing support: This work has not received any contribution, grant or scholarship.

Confidentiality of data: The authors declare that they have followed the protocols of their work center on the publication of data from patients.

Patient Consent: Consent for publication was obtained.

Provenance and peer review: Not commissioned; externally peer reviewed

ORCID

Allen de Souza Pessoa

https://orcid.org/0000-0002-0608-8918

Juliana Câmara Mariz

https://orcid.org/0000-0002-7292-1648

Bárbara de Oliveira Martins

https://orcid.org/0000-0002-6650-1719

Laura Franco Belga

https://orcid.org/0000-0002-8355-4185

Evelyne Mara Costa

https://orcid.org/0000-0002-0159-626X

Daniel Lago Obadia

https://orcid.org/0000-0001-9536-1615 


\section{Caso Clínico}

\section{REFERÊNCIAS}

1. Victory R, Diamond MP, Johns DA. Villar's nodule: a case report and systematic literature review of endometriosis externa of the umbilicus. J Minim Invasive Gynecol. 2007;14:23-32. doi:10.1016/i.jmig.2006.07.014

2. Calagna G, Perino A, Chianetta D, Vinti D, Triolo $M M$, Rimi $C$, et al. Primary umbilical endometrioma: analyzing the pathogenesis of endometriosis from an unusual localization. Taiwan J Obstet Gynecol. 2015; 54:306-12. doi:10.1016/i.tjog.2014.03.011

3. Fernández-Aceñero MJ, Córdova $\mathrm{S}$. Cutaneous endometriosis: review of 15 cases diagnosed at a single institution. Arch Gynecol Obstet. 2010; 283:1041-4. doi:10.1007/s00404-010-1484-3

4. Kahlenberg LK, Laskey S. Primary umbilical endometriosis presenting as umbilical drainage in a nulliparous and surgically naive young woman. Am J Emerg Med. 2014;32:692.e1-692.e2. doi:10.1016/i. ajem.2013.12.024

5. FernándezVozmedianoJM,ArmarioHitaJC, CuevasSantosJ. Cutaneous endometriosis. Int J Dermatol. 2010;49:1410-
2. doi: $10.1111 / j .1365-4632.2010 .04585 . x$

6. Lopez-Soto A, Sanchez-Zapata MI, Martinez-Cendan JP, Ortiz Reina S, Bernal Mañas CM, Remezal Solano M. Cutaneous endometriosis: Presentation of 33 cases and literature review. Eur J Obstet Gynecol Reprod Biol. 2018;221:58-63. doi:10.1016/j.ejogrb.2017.11.024

7. Chatzikokkinou P, Thorfinn J, Angelidis IK, Papa G, Trevisan $G$. Spontaneous endometriosis in an umbilical skin lesion. Acta Dermatovenerol Alp Pannonica Adriat. 2009; 18:126-30.

8. Boesgaard-Kjer D, Boesgaard-Kjer D, Kjer JJ. Primary umbilical endometriosis (PUE). Eur J Obstet Gynecol Reprod Biol. 2017;209:44-45. doi:10.1016/i. ejogrb.2016.05.030

9. Loh SH, Lew BL, Sim WY. Primary Cutaneous Endometriosis of Umbilicus. Ann Dermatol. 2017; 29:621-5. doi: 10.5021/ad.2017.29.5.621

10. Fancellu A, Pinna A, Manca A, Capobianco G, Porcu A. Primary umbilical endometriosis. Case report and discussion on management options. Int J Surg Case Rep. 2013; 4:1145-8. doi:10.1016/i.ijscr.2013.11.001 